\title{
Generalized Well-Posedness for Symmetric Vector Quasi-Equilibrium Problems
}

\author{
Wei-bing Zhang, ${ }^{1}$ Nan-jing Huang, ${ }^{1}$ and Donal O'Regan ${ }^{2,3}$ \\ ${ }^{1}$ Department of Mathematics, Sichuan University, Chengdu 610064, China \\ ${ }^{2}$ School of Mathematics, Statistics and Applied Mathematics, National University of Ireland, Galway, Ireland \\ ${ }^{3}$ NAAM Research Group, Department of Mathematics, King Abdulaziz University, Jeddah, Saudi Arabia
}

Correspondence should be addressed to Nan-jing Huang; nanjinghuang@hotmail.com

Received 12 August 2014; Accepted 23 September 2014

Academic Editor: Wei-Shih Du

Copyright (C) 2015 Wei-bing Zhang et al. This is an open access article distributed under the Creative Commons Attribution License, which permits unrestricted use, distribution, and reproduction in any medium, provided the original work is properly cited.

We introduce and study well-posedness in connection with the symmetric vector quasi-equilibrium problem, which unifies its Hadamard and Levitin-Polyak well-posedness. Using the nonlinear scalarization function, we give some sufficient conditions to guarantee the existence of well-posedness for the symmetric vector quasi-equilibrium problem.

\section{Introduction}

Vector equilibrium problem provides a very general model for many problems like the vector variational inequality problem, the vector complementarity problem, the vector optimization problem, the multiobjective game problem, the vector network equilibrium problem, and the vector saddle point problem (see, e.g., [1-3] and the references therein).

Well-posedness plays an important role in the theory and numerical methods for optimization. The first concept of well-posedness was introduced by Tykhonov [4] for a global minimization problem having a unique solution. Lucchetti and Patrone [5] introduced the notion of wellposedness for variational inequalities. Lignola and Morgan [6] studied the well-posedness of optimization problems with variational inequality constraints. Fang et al. [7] investigated well-posedness for equilibrium problems and optimization problems with equilibrium constraints. Hu et al. [8] studied well-posedness of systems of equilibrium problems.

The notions of well-posedness can be mainly divided into three groups, namely, Hadamard type, Tykhonov type, and Levitin-Polyak type. Researchers have studied the relations between the Hadamard well-posedness and Tykhonov wellposedness for different problems (see $[9,10]$ ). Most of the literature deals with directly specific notions of wellposedness. Huang et al. [11] investigated the Levitin-Polyak well-posedness of variational inequalities problems with functional constraints. S. J. Li and M. H. Li [12] studied the Levitin-Polyak well-posedness of vector equilibrium problems. Li et al. [13] investigated Levitin-Polyak wellposedness of generalized vector quasi-equilibrium problems. Peng et al. [14] studied Levitin-Polyak well-posedness of generalized vector equilibrium problems with both abstract set constraints and functional constraints. Salamon [15] considered the Hadamard well-posedness by using the vector topological pseudomonotonicity. Peng et al. [16] investigated the Hadamard well-posedness of vector equilibrium problems by considering the perturbations of both vector-valued functions and feasible sets. Li and Zhang [17] studied the Hadamard well-posedness for the vector-valued optimization problems. Long and Huang [18] considered the $\alpha$-well posedness for the symmetric quasi-equilibrium problems. Recently, Han and Gong [19] studied the generalized LevitinPolyak well-posedness of symmetric strong vector quasiequilibrium problems. Deng and Xiang [20] introduced and studied the generalized well-posedness for the generalized vector equilibrium, which unifies its Hadamard and LevitinPolyak well-posedness.

Motivated and inspired by the papers mentioned above, in this paper, we introduce a well-posedness concept for the symmetric vector quasi-equilibrium problem, which unifies its Hadamard and Levitin-polyak well-posedness. By 
employing the scalarization function, we give some sufficient conditions to guarantee the existence of the well-posedness for the symmetric vector quasi-equilibrium problem in real locally convex Hausdorff topological vector spaces. The results presented in this paper generalize and extend Theorem 4.2 of [16] and Theorem 4.1 of [20].

\section{Preliminaries}

Let $X$ and $Y$ be nonempty subsets of real locally convex Hausdorff topological vector spaces $F$ and $W$, respectively. Assume $S: X \times Y \rightrightarrows X$ and $G: X \times Y \rightrightarrows Y$ are two set-valued mappings. Let $Z$ be a real topological vector space and $C \subset Z$ be a closed convex pointed cone with int $C \neq \emptyset$, where int $C$ denotes the topological interior of $C$. It is well known that the cone $C$ can induce the following orders:

$$
\begin{gathered}
z_{1} \leq z_{2} \quad \text { iff } z_{2}-z_{1} \in C, \\
z_{1}<z_{2} \quad \text { iff } z_{2}-z_{1} \in \operatorname{int} C .
\end{gathered}
$$

Let $\varphi: X \times X \times Y \rightarrow Z$ and $\psi: X \times Y \times Y \rightarrow Z$ be two vector valued functions. Let $C_{1}$ and $C_{2}$ be two closed convex pointed cones of $Z$ with int $C_{1} \neq \emptyset$ and int $C_{2} \neq \emptyset$. In this paper, we consider the following the symmetric vector quasiequilibrium problem (in short SVQEP): find $\left(x_{0}, y_{0}\right) \in X \times Y$ such that $x_{0} \in S\left(x_{0}, y_{0}\right), y_{0} \in G\left(x_{0}, y_{0}\right)$, and

$$
\begin{array}{ll}
\varphi\left(x_{0}, u, y_{0}\right) \notin-\operatorname{int} C_{1}, & \forall u \in S\left(x_{0}, y_{0}\right), \\
\psi\left(x_{0}, v, y_{0}\right) \notin-\operatorname{int} C_{2}, & \forall v \in G\left(x_{0}, y_{0}\right) .
\end{array}
$$

Some special cases of SVQEP are as follows.

(I) If $C_{1}=C_{2}=C, \varphi(x, u, y)=f(u, y)-f(x, y)$, and $\psi(x, v, y)=g(x, v)-g(x, y)$ for all $(x, y) \in X \times Y$, where $f, g: X \times Y \rightarrow Z$ are two mappings, then (SVQEP) reduces to the problem of finding $\left(x_{0}, y_{0}\right) \in X \times Y$ such that $x_{0} \in$ $S\left(x_{0}, y_{0}\right), y_{0} \in G\left(x_{0}, y_{0}\right)$, and

$$
\begin{aligned}
& f\left(x, y_{0}\right)-f\left(x_{0}, y_{0}\right) \notin-\operatorname{int} C, \quad \forall x \in S\left(x_{0}, y_{0}\right), \\
& g\left(x_{0}, y\right)-g\left(x_{0}, y_{0}\right) \notin-\operatorname{int} C, \quad \forall x \in G\left(x_{0}, y_{0}\right) .
\end{aligned}
$$

This problem was studied in Fu [21] and Han and Gong [19].

(II) If $Z=(-\infty,+\infty)$ and $C_{1}=C_{2}=[0,+\infty)$, then (SVQEP) reduces to the following problem: find $\left(x_{0}, y_{0}\right) \in$ $X \times Y$ such that $x_{0} \in S\left(x_{0}, y_{0}\right), y_{0} \in G\left(x_{0}, y_{0}\right)$, and

$$
\begin{gathered}
\varphi\left(x_{0}, u, y_{0}\right) \geq 0, \quad \forall u \in S\left(x_{0}, y_{0}\right), \\
\psi\left(x_{0}, v, y_{0}\right) \geq, \quad \forall v \in G\left(x_{0}, y_{0}\right) .
\end{gathered}
$$

(III) If $Z=(-\infty,+\infty), C_{1}=C_{2}=[0,+\infty), \varphi(x, u, y)=$ $f(u, y)-f(x, y)$, and $\psi(x, v, y)=g(x, v)-g(x, y)$ for all $(x, y) \in X \times Y$, where $f, g: X \times Y \rightarrow Z$ are two mappings, then (SVQEP) reduces to the symmetric quasi-equilibrium problem: find $\left(x_{0}, y_{0}\right) \in X \times Y$ such that $x_{0} \in S\left(x_{0}, y_{0}\right), y_{0} \in$ $G\left(x_{0}, y_{0}\right)$, and

$$
\begin{aligned}
& f\left(x, y_{0}\right) \geq f\left(x_{0}, y_{0}\right), \quad \forall x \in S\left(x_{0}, y_{0}\right), \\
& g\left(x_{0}, y\right) \geq g\left(x_{0}, y_{0}\right), \quad \forall x \in G\left(x_{0}, y_{0}\right) .
\end{aligned}
$$

This problem was considered in Long and Huang [18].
It is well known that SVQEP includes many important problems as special cases, such as equilibrium problems, Nash equilibrium problems, quasivariational inequalities, variational inequalities, and fixed point problems.

Now we recall some useful definitions and lemmas.

Let $(X, d)$ be a metric space. Denote a family of all nonempty compact subsets of $X$ by $K(X)$. For any $A, B \in$ $K(X)$, let

$$
H(A, B)=\max \left\{\sup _{a \in A} \inf _{b \in B} d(a, b), \sup _{b \in B} \inf _{a \in A} d(a, b)\right\}
$$

denote the Hausdorff metric on $K(X)$. It is well known that $(K(X), H)$ is complete if and only if $(X, d)$ is complete.

Definition 1. A sequence $\left\{\left(x_{n}, y_{n}\right)\right\} \subset X \times Y$ is called a LevitinPolyak (in short LP) approximating solution sequence converging to $\left(x_{0}, y_{0}\right)$ for SVQEP, if there exists $\varepsilon_{n}>0$ with $\varepsilon_{n} \rightarrow 0$ such that

$$
\begin{aligned}
& d\left(x_{n}, S\left(x_{n}, y_{n}\right)\right) \leq \varepsilon_{n}, \quad d\left(y_{n}, G\left(x_{n}, y_{n}\right)\right) \leq \varepsilon_{n}, \\
& \varphi\left(x_{0}, u, y_{0}\right)+\varepsilon_{n} e_{1} \notin-\operatorname{int} C_{1}, \quad \forall u \in S\left(x_{0}, y_{0}\right), \\
& \psi\left(x_{0}, v, y_{0}\right)+\varepsilon_{n} e_{2} \notin-\operatorname{int} C_{2}, \quad \forall v \in G\left(x_{0}, y_{0}\right),
\end{aligned}
$$

where $e_{1} \in \operatorname{int} C_{1}$ and $e_{2} \in \operatorname{int} C_{2}$ are given points.

Definition 2 (see [3]). Let $E, Z$ be two real Hausdorff topological spaces, $X \subset E$ a nonempty subset, and $C \subset Z$ a closed convex pointed cone. A mapping $F: X \rightarrow Z$ is said to be

(i) $C$-upper semicontinuous (C-u.s.c.) (resp., $C$-lower semicontinuous (C-l.s.c.) at $x_{0} \in X$ if, for any neighborhood $V$ of zero in $Z$, there exists a neighborhood $U$ of zero in $E$ such that

$$
\begin{aligned}
& F(x) \in F\left(x_{0}\right)+V-C, \quad \forall x \in U \cap X, \\
& \left(\text { resp., } F(x) \in F\left(x_{0}\right)+V+C, \forall x \in U \cap X\right) .
\end{aligned}
$$

(ii) C-u.s.c. (resp., C-l.s.c.) on $X$ if it is $C$-u.s.c. (resp., $C$ l.s.c.) at every point $x \in X$.

Definition 3 (see [22]). Let $X$ and $Y$ be two topological spaces. A set-valued mapping $F: X \rightrightarrows Y$ is said to be

(i) upper semicontinuous (u.s.c.) at $x_{0}$ if, for any open set $V \supset F\left(x_{0}\right)$, there is an open neighborhood $O_{x_{0}}$ of $x_{0}$ such that $F\left(x^{\prime}\right) \subset V$ for each $x^{\prime} \in O_{x_{0}}$,

(ii) lower semicontinuous (1.s.c.) at $x_{0}$ if, for any open set $V \cap F\left(x_{0}\right) \neq \emptyset$, there is an open neighborhood $O_{x_{0}}$ of $x_{0}$ such that $F\left(x^{\prime}\right) \cap V \neq \emptyset$ for each $x^{\prime} \in O_{x_{0}}$,

(iii) continuous at $x_{0}$ if it is both upper and lower semicontinuous at $x_{0}$,

(iv) upper semicontinuous (lower semicontinuous or continuous) on $X$ if it is upper semicontinuous (lower semicontinuous or continuous) at every $x \in X$, 
(v) an usco mapping if $F$ is upper semicontinuous on $X$ and $F(x)$ is compact for each $x \in X$,

(vi) closed if and only if its graph $\operatorname{Graph}(F):=\{(x, y) \in$ $X \times Y: y \in F(x)\}$ is closed.

Lemma 4 (see [23]). Let $X$ and $Y$ be two topological spaces and $F: X \rightrightarrows Y$ a set-valued mapping. Suppose that $Y$ is compact. Then $F$ is closed if and only if it is upper semicontinuous.

Lemma 5 (see [24]). Let $A$ and $A_{n}(n=1,2, \ldots)$ all be nonempty compact subsets of a metric space $X$ with $A_{n} \rightarrow A$ in the Hausdorff metric topology. Then the following statements hold.

(i) $\bigcup_{n=1}^{+\infty} A_{n} \cup A$ is also nonempty compact subset of $X$.

(ii) If $x_{n} \in A_{n}, x_{n} \rightarrow x$, then $x \in A$.

(iii) For any $x \in A$, there exists $x_{n} \in A_{n}$ such that $x_{n} \rightarrow x$.

Lemma 6 (see [25]). Let $X$ and $Y$ be two topological spaces and $F: X \rightrightarrows Y$ be an usco mapping. Then for any net $\left\{x_{\alpha}\right\} \subset X$ with $x_{\alpha} \rightarrow x$ and $y_{\alpha} \in F\left(x_{\alpha}\right)$, there exists a subnet $\left\{y_{\alpha^{\prime}}\right\} \subset y_{\alpha}$ such that $y_{\alpha^{\prime}} \rightarrow y \in F(x)$.

Lemma 7 (see [26]). For any fixed point $e \in \operatorname{int} C$, the nonlinear scalarization function $\xi_{e}: Y \rightarrow \mathbb{R}$ is defined by

$$
\xi_{e}(y)=\inf \{r \in \mathbb{R}: y \in r e-C\}, \quad \forall y \in Y .
$$

The nonlinear scalarization function $\xi_{e}$ has the following properties:

(i) $\xi_{e}(y)<r \Leftrightarrow y \in r e-\operatorname{int} C$,

(ii) $\xi_{e}(y) \leq r \Leftrightarrow y \in r e-C$,

(iii) $\xi_{e}(y) \geq r \Leftrightarrow y \notin r e-\operatorname{int} C$,

(iv) $\xi_{e}(y)>r \Leftrightarrow y \notin r e-C$,

(v) $\xi_{e}(y)=r \Leftrightarrow y \in r e-\partial C$, where $\partial C$ denotes the topological boundary of $C$.

Lemma 8. If $\varphi: X \times X \times Y \rightarrow Z$ is $C$-upper semicontinuous, then $\xi_{e} \varphi$ is upper semicontinuous.

Proof. For any fixed $r \in \mathbb{R}=(-\infty,+\infty)$, let

$$
B=\left\{(x, u, y) \in X \times X \times Y: \xi_{e}(\varphi(x, u, y)) \geq r\right\} .
$$

In order to show that $\xi_{e} \varphi$ is upper semicontinuous, we only need to show that $B$ is closed. Letting $\left(x_{n}, u_{n}, y_{n}\right) \in B$ with $\left(x_{n}, u_{n}, y_{n}\right) \rightarrow(x, u, y)$, then

$$
\xi_{e} \varphi\left(x_{n}, u_{n}, y_{n}\right) \geq r
$$

and Lemma 7 shows that

$$
\varphi\left(x_{n}, u_{n}, y_{n}\right) \notin r e-\operatorname{int} C .
$$

Suppose to the contrary that $(x, u, y) \notin B$. We have

$$
\xi_{e}(\varphi(x, u, y))<r .
$$

It follows from Lemma 7 that

$$
\varphi(x, u, y)-r e \epsilon-\operatorname{int} C .
$$

This implies that there exists a neighborhood $V$ of zero element in $Z$ such that

$$
\varphi(x, u, y)-r e+V \in-\operatorname{int} C .
$$

Since $\varphi$ is $C$-upper semicontinuous, for the above $V$, there exists a positive integral number $N$ such that, when $n>N$, one has

$$
\varphi\left(x_{n}, u_{n}, y_{n}\right) \in \varphi(x, u, y)+V-C
$$

From (15) and (16), when $n>N$, we have

$$
\begin{aligned}
\varphi\left(x_{n}, u_{n}, y_{n}\right) & \in \varphi(x, u, y)+V-C \\
& \subset r e-\operatorname{int} C-C \\
& \subset r e-\operatorname{int} C
\end{aligned}
$$

which is in contradiction with (12). Thus, $(x, u, y) \in B$ and so $\xi_{e} \varphi$ is upper semicontinuous. This completes the proof.

Example 9. Let $X=Y=\mathbb{R}=(-\infty,+\infty)$ and $C=[0,+\infty)$. Let

$$
\varphi(x, y, z)= \begin{cases}2, & \text { if } x=y=z=1 \\ x+y-z, & \text { otherwise }\end{cases}
$$

Then it is easy to check that $\varphi$ is $C$-upper semicontinuous and so Lemma 8 shows that $\xi_{e} \varphi$ is upper semicontinuous for any $e \in \operatorname{int} C$.

\section{Bounded Rationality Model and Definition of Well-Posedness for SVQEP}

Let $\left(X, d_{1}\right)$ and $\left(Y, d_{2}\right)$ be two metric spaces and $Z$ be a Banach space with a norm $\|\cdot\|$. Let $C_{1}$ and $C_{2}$ be nonempty closed convex pointed cones of $Z$ with apex at the origin such that $\operatorname{int} C_{1} \neq \emptyset$ and int $C_{2} \neq \emptyset$.

In this section, we first define the problem space $\Lambda$ of SVQEP as follows: $\Lambda=\{(\varphi, \psi, S, G): \varphi: X \times X \times Y \rightarrow$ $Z$ is $C_{1}$-upper semicontinuous on $X \times X \times Y, \psi$ : $X \times Y \times Y \rightarrow Z$ is $C_{2}$-upper semicontinuous on $X \times$ $Y \times Y, \varphi(x, x, y)=0$ and $\psi(x, y, y)=$ 0 for all $(x, y) \in X \times Y, \sup _{(x, u, y) \in X \times X \times Y}\|\varphi(x, u, y)\|<$ $+\infty \sup _{(x, v, y) \in X \times Y \times Y}\|\psi(x, v, y)\|<+\infty, S: X \times Y \rightrightarrows X$ and $G: X \times Y \rightrightarrows Y$ are continuous with compact values, and there exists $(x, y) \in X \times Y$ such that $x \in S(x, y), y \in G(x, y)$ and $\varphi(x, u, y) \notin-\operatorname{int} C_{1}, \psi(x, v, y) \notin-\operatorname{int} C_{2}$ for all $u \in S(x, y)$ and $v \in G(x, y)\}$. 
For any $\lambda_{i}=\left(\varphi_{i}, \psi_{i}, S_{i}, G_{i}\right) \in \Lambda$ with $i=1,2$, define

$$
\begin{aligned}
\rho\left(\lambda_{1}, \lambda_{2}\right)= & \sup _{(x, u, y) \in X \times X \times Y}\left\|\varphi_{1}(x, u, y)-\varphi_{2}(x, u, y)\right\| \\
& +\sup _{(x, v, y) \in X \times Y \times Y}\left\|\psi_{1}(x, v, y)-\psi_{2}(x, v, y)\right\| \\
& +\sup _{(x, y) \in X \times Y} H_{1}\left(S_{1}(x, y), S_{2}(x, y)\right) \\
& +\sup _{(x, y) \in X \times Y} H_{2}\left(G_{1}(x, y), G_{2}(x, y)\right)
\end{aligned}
$$

where $H_{1}$ and $H_{2}$ denote the Hausdorff metric on $K(X)$ and $K(Y)$, respectively. Then it is easy to see that $(\Lambda, \rho)$ is a metric space.

Next we define the bounded rationality model $M=$ $\{\Lambda, X, Y, f, \Phi\}$ for (SVQEP) as follows.

(i) $(\Lambda, \rho),\left(X, d_{1}\right)$ and $\left(Y, d_{2}\right)$ are metric spaces.

(ii) The feasible set of the symmetric vector quasiequilibrium problems $\lambda \in \Lambda$ is defined by

$f(\lambda):=\{(x, y) \in X \times Y: x \in S(x, y), y \in G(x, y)\}$.

(iii) The solution set of problem $\lambda \in \Lambda$ is defined by

$$
\begin{aligned}
E(\lambda)= & \{(x, y) \in X \times Y:(x, y) \in f(\lambda) \text { such that } \\
& \varphi(x, u, y) \notin-\operatorname{int} C_{1} \forall u \in S(x, y), \\
& \left.\psi(x, v, y) \notin-\operatorname{int} C_{2} \quad \forall v \in G(x, y)\right\} .
\end{aligned}
$$

(iv) For any $(x, y) \in X \times Y$, define

$d((x, y), f(\lambda))=d_{1}(x, S(x, y))+d_{2}(y, G(x, y))$.

(v) The rationality function of the problem $\lambda \in \Lambda$ is defined by

$$
\begin{aligned}
& \Phi(\lambda,(x, y)) \\
& =\max \left\{\sup _{u \in S(x, y)}-\xi_{e_{1}} \varphi(x, u, y), \sup _{v \in G(x, y)}-\xi_{e_{2}} \psi(x, v, y)\right\},
\end{aligned}
$$

where $e_{1} \in \operatorname{int} C_{1}$ and $e_{2} \in \operatorname{int} C_{2}$.

Lemma 10. (i) For any $\lambda \in \Lambda$ and $(x, y) \in f(\lambda)$, $\Phi(\lambda,(x, y)) \geq 0$.

(ii) For any $\lambda \in \Lambda, E(\lambda) \neq \emptyset$.

(iii) For $\lambda \in \Lambda$ and $\epsilon \geq 0$ with $(x, y) \in f(\lambda), \Phi(\lambda,(x, y)) \leq$ $\epsilon$ if and only if

$$
\begin{array}{ll}
\varphi(x, u, y)+\epsilon e_{1} \notin-\operatorname{int} C_{1}, & \forall u \in S(x, y), \\
\psi(x, v, y)+\epsilon e_{2} \notin-\operatorname{int} C_{2}, & \forall v \in G(x, y) .
\end{array}
$$

In particular, $(x, y) \in E(\lambda)$ if and only if $(x, y) \in f(\lambda)$ and $\Phi(\lambda,(x, y))=0$.
Proof. (i) For any $\lambda \in \Lambda$ and $(x, y) \in f(\lambda)$, we have $x \in$ $S(x, y)$ and $y \in G(x, y)$. From Lemma $7(\mathrm{v})$, we know that $\xi_{e_{1}} \varphi(x, x, y)=0$ and $\xi_{e_{2}} \psi(x, y, y)=0$, since $\varphi(x, x, y)=0$ and $\psi(x, y, y)=0$. Thus, one has

$$
\begin{aligned}
& \Phi(\lambda,(x, y)) \\
& =\max \left\{\sup _{u \in S(x, y)}-\xi_{e_{1}} \varphi(x, u, y), \sup _{v \in G(x, y)}-\xi_{e_{2}} \psi(x, v, y)\right\} \\
& \geq \max \left\{-\xi_{e_{1}} \varphi(x, x, y),-\xi_{e_{2}} \psi(x, y, y)\right\}=0 .
\end{aligned}
$$

(ii) It is obvious that $E(\lambda) \neq \emptyset$ for all $\lambda \in \Lambda$.

(iii) Assume that $\lambda \in \Lambda$ and $\epsilon \geq 0$ such that $\Phi(\lambda,(x, y)) \leq$ $\epsilon$. Then we have

$$
\max \left\{\sup _{u \in S(x, y)}-\xi_{e_{1}} \varphi(x, u, y), \sup _{v \in G(x, y)}-\xi_{e_{2}} \psi(x, v, y)\right\} \leq \epsilon
$$

and so

$$
\sup _{u \in S(x, y)}-\xi_{e_{1}} \varphi(x, u, y) \leq \epsilon
$$

with

$$
\sup _{v \in G(x, y)}-\xi_{e_{2}} \psi(x, v, y) \leq \epsilon \text {. }
$$

It follows that

$$
\begin{aligned}
& \xi_{e_{1}} \varphi(x, u, y) \geq-\epsilon, \quad \forall u \in S(x, y), \\
& \xi_{e_{2}} \psi(x, v, y) \geq-\epsilon, \quad \forall v \in G(x, y) .
\end{aligned}
$$

By Lemma 7(iii), one has

$$
\begin{array}{ll}
\varphi(x, u, y)+\epsilon e_{1} \notin-\operatorname{int} C_{1}, & \forall u \in S(x, y), \\
\psi(x, v, y)+\epsilon e_{2} \notin-\operatorname{int} C_{2}, & \forall v \in G(x, y) .
\end{array}
$$

Conversely, assume that $\lambda \in \Lambda$ and $\epsilon \geq 0$ with $(x, y) \epsilon$ $f(\lambda)$ such that

$$
\begin{aligned}
& \varphi(x, u, y)+\epsilon e_{1} \notin-\operatorname{int} C_{1}, \quad \forall u \in S(x, y), \\
& \psi(x, v, y)+\epsilon e_{2} \notin-\operatorname{int} C_{2}, \quad \forall v \in G(x, y) .
\end{aligned}
$$

Then Lemma 7(iii) shows that

$$
\begin{aligned}
& \xi_{e_{1}} \varphi(x, u, y) \geq-\epsilon, \quad \forall u \in S(x, y), \\
& \xi_{e_{2}} \varphi(x, v, y) \geq-\epsilon, \quad \forall v \in G(x, y) .
\end{aligned}
$$

These inequalities imply that

$$
\begin{gathered}
\sup _{u \in S(x, y)}-\xi_{e_{1}} \varphi(x, u, y) \leq \epsilon, \\
\sup _{v \in G(x, y)}-\xi_{e_{2}} \psi(x, v, y) \leq \epsilon .
\end{gathered}
$$


Therefore, we get

$$
\begin{aligned}
& \Phi(\lambda,(x, y)) \\
& =\max \left\{\sup _{u \in S(x, y)}-\xi_{e_{1}} \varphi(x, u, y), \sup _{v \in G(x, y)}-\xi_{e_{2}} \psi(x, v, y)\right\} \\
& \leq \epsilon .
\end{aligned}
$$

This completes the proof.

Remark 11. Lemma 10 is a generalization of Lemma 3.1 of Deng and Xiang [20].

Example 12. Let $X=Y=(-\infty,+\infty), C_{1}=C_{2}=C=$ $[0,+\infty)$, and $e_{1}=e_{2}=1$. Assume that

$$
\begin{gathered}
S(x, y)=G(x, y)=[0,1], \quad \forall x, y \in X \times Y, \\
\varphi(x, u, y)=x-u, \quad \psi(x, v, y)=y-v, \\
\forall(x, y) \in X \times Y, \quad u \in S(x, y), \quad v \in G(x, y) .
\end{gathered}
$$

Then it is easy to see that

$$
\begin{gathered}
f(\lambda)=\{(x, y): x \in[0,1], y \in[0,1]\}, \\
\Phi(\lambda,(x, y)) \\
=\max \left\{\sup _{u \in[0,1]}-\xi_{1}(x-u), \sup _{v \in[0,1]}-\xi_{1}(y-v)\right\} .
\end{gathered}
$$

(i) If $(x, y) \in f(\lambda)$, then $x \in[0,1]$ and $y \in[0,1]$. It is obvious that

$$
\Phi(\lambda,(x, y)) \geq 0
$$

(ii) For any $\lambda \in \Lambda$, one has

$$
E(\lambda)=\{(x, y): x=1, y=1\} \neq \emptyset .
$$

(iii) It is easy to check that $(x, y) \in E(\lambda)$ if and only if $\Phi(\lambda,(x, y))=0$. Moreover, taking $x=0, y=1 / 2$, then $(x, y)=(0,1 / 2) \notin E(\lambda)$ and $\Phi(\lambda,(x, y))=1 \neq$ 0 .

By Definition 1 and Lemma 10, for all $\varepsilon_{n}>0$ with $\varepsilon_{n} \rightarrow 0$, the LP approximating solution set for the problem $\lambda$ is defined as

$$
\begin{gathered}
E\left(\lambda, \varepsilon_{n}\right)=\left\{(x, y) \in X \times Y: d((x, y), f(\lambda)) \leq \varepsilon_{n},\right. \\
\left.\Phi(\lambda,(x, y)) \leq \varepsilon_{n}\right\},
\end{gathered}
$$

and the set of solution for the problem $\lambda$ is defined as

$$
\begin{aligned}
E(\lambda) & =E(\lambda, 0) \\
& =\{(x, y) \in X \times Y:(x, y) \in f(\lambda), \Phi(\lambda,(x, y))=0\} .
\end{aligned}
$$

Next we define the Levitin-Polyak well-posedness and the Hadamard well-posedness for (SVQEP) as follows.
Definition 13. (i) If, for any $\left(x_{n}, y_{n}\right) \in E\left(\lambda, \varepsilon_{n}\right), \varepsilon_{n}>0$ with $\varepsilon_{n} \rightarrow 0$, there exists a subsequence $\left\{\left(x_{n_{k}}, y_{n_{k}}\right)\right\} \subset\left\{\left(x_{n}, y_{n}\right)\right\}$ such that $\left(x_{n_{k}}, y_{n_{k}}\right) \rightarrow(x, y) \in E(\lambda)$, then the problem $\lambda \in \Lambda$ is said to be generalized LP well-posedness.

(ii) If $E(\lambda)=\{(x, y)\}$ (a singleton), for any $\left(x_{n}, y_{n}\right) \in$ $E\left(\lambda, \varepsilon_{n}\right), \varepsilon_{n}>0$ with $\varepsilon_{n} \rightarrow 0$, there exists a subsequence $\left\{\left(x_{n_{k}}, y_{n_{k}}\right)\right\} \subset\left\{\left(x_{n}, y_{n}\right)\right\}$ such that $\left(x_{n_{k}}, y_{n_{k}}\right) \rightarrow(x, y) \in E(\lambda)$, then the problem $\lambda \in \Lambda$ is said to be LP well-posedness.

Definition 14. (i) If, for any $\lambda_{n} \in \Lambda$ with $\lambda_{n} \rightarrow \lambda$ and any $\left(x_{n}, y_{n}\right) \in E\left(\lambda_{n}\right)$, there exists a subsequence $\left\{\left(x_{n_{k}}, y_{n_{k}}\right)\right\} \subset$ $\left\{\left(x_{n}, x_{n}\right)\right\}$, such that $\left(x_{n_{k}}, y_{n_{k}}\right) \rightarrow(x, y) \in E(\lambda)$, then the problem $\lambda \in \Lambda$ is said to be generalized Hadamard wellposed.

(ii) If $E(\lambda)=\{(x, y)\}$ (a singleton), for any $\lambda_{n} \in \Lambda$ with $\lambda_{n} \rightarrow \lambda$ and any $\left(x_{n}, y_{n}\right) \in E\left(\lambda_{n}\right)$, we have $\left(x_{n}, y_{n}\right) \rightarrow$ $(x, y) \in E(\lambda)$, then the problem $\lambda \in \Lambda$ is said to be Hadamard well-posed.

By Definitions 13 and 14, we introduce the definition of (generalized) well-posedness, which unifies its LevitinPolyak well-posedness and Hadamard well-posedness.

Definition 15. (i) If for any $\lambda_{n} \in \Lambda$ with $\lambda_{n} \rightarrow \lambda,\left(x_{n}, y_{n}\right) \in$ $E\left(\lambda_{n}, \varepsilon_{n}\right), \varepsilon_{n}>0$ with $\varepsilon_{n} \rightarrow 0$, there exists a subsequence $\left\{\left(x_{n_{k}}, y_{n_{k}}\right)\right\} \subset\left\{\left(x_{n}, y_{n}\right)\right\}$ such that $\left(x_{n_{k}}, y_{n_{k}}\right) \rightarrow(x, y) \in$ $E(\lambda)$, then the problem $\lambda \in \Lambda$ is said to be generalized well-posed.

(ii) If $E(\lambda)=\{(x, y)\}$ (a singleton), for any $\lambda_{n} \in \Lambda$ with $\lambda_{n} \rightarrow \lambda,\left(x_{n}, y_{n}\right) \in E\left(\lambda_{n}, \varepsilon_{n}\right), \varepsilon_{n}>0$ with $\varepsilon_{n} \rightarrow 0$, we have $\left(x_{n}, y_{n}\right) \rightarrow(x, y) \in E(\lambda)$, then the problem $\lambda \in \Lambda$ is said to be well-posed.

Lemma 16. If the problem $\lambda \in \Lambda$ is (generalized) well-posed, then

(i) the problem $\lambda \in \Lambda$ must be (generalized) LP well-posed;

(ii) the problem $\lambda \in \Lambda$ must be (generalized) Hadamard well-posed.

Proof. We only prove the statement of generalized wellposedness. The proof of the well-posedness is similar to the generalized well-posedness.

(i) For any $\left(x_{n}, y_{n}\right) \in E\left(\lambda, \varepsilon_{n}\right)$, letting $\lambda_{n}=\lambda$, we know that $\lambda_{n} \rightarrow \lambda$ and $\left(x_{n}, y_{n}\right) \in E\left(\lambda_{n}, \varepsilon_{n}\right)$. Since the problem $\lambda \in \Lambda$ is generalized well-posed, there exists a subsequence $\left\{\left(x_{n_{k}}, y_{n_{k}}\right)\right\} \subset\left\{\left(x_{n}, y_{n}\right)\right\}$ such that $\left(x_{n_{k}}, y_{n_{k}}\right) \rightarrow$ $(x, y) \in E(\lambda)$. Thus, it follows that $\lambda$ is generalized LP well-posed.

(ii) For any $\lambda_{n} \in \Lambda$ with $\lambda_{n} \rightarrow \lambda$ and $\left(x_{n}, y_{n}\right) \in E\left(\lambda_{n}\right)$, letting $\varepsilon_{n}=0$, it is easy to see that $\left(x_{n}, y_{n}\right) \in E\left(\lambda_{n}, \varepsilon_{n}\right)$. Since the problem $\lambda \in \Lambda$ is generalized well-posed, there exists a subsequence $\left\{\left(x_{n_{k}}, y_{n_{k}}\right)\right\} \subset\left\{\left(x_{n}, y_{n}\right)\right\}$ such that $\left(x_{n_{k}}, y_{n_{k}}\right) \rightarrow$ $(x, y) \in E(\lambda)$. Therefore, we know that $\lambda$ is generalized Hadamard well-posed.

This completes the proof. 


\section{Some Sufficient Conditions for Well-Posedness of SVQEP}

Assume $\left(X, d_{1}\right)$ and $\left(Y, d_{2}\right)$ are compact metric spaces. In this section, we give some sufficient conditions to guarantee the existence of the well-posedness for SVQEP.

Lemma 17. $(\Lambda, \rho)$ is a complete metric space.

Proof. Let $\left\{\lambda_{n}=\left(\varphi_{n}, \psi_{n}, S_{n}, G_{n}\right)\right\}$ be a Cauchy sequence in $\Lambda$. Then for any given $\varepsilon>0$, when integral numbers $m$ and $n$ are large enough, we have

$$
\begin{aligned}
\rho\left(\lambda_{n}, \lambda_{m}\right)= & \sup _{(x, u, y) \in X \times X \times Y}\left\|\varphi_{n}(x, u, y)-\varphi_{m}(x, u, y)\right\| \\
& +\sup _{(x, v, y) \in X \times Y \times Y}\left\|\psi_{n}(x, v, y)-\psi_{m}(x, v, y)\right\| \\
& +\sup _{(x, y) \in X \times Y} H_{1}\left(S_{n}(x, y), S_{m}(x, y)\right) \\
& +\sup _{(x, y) \in X \times Y} H_{2}\left(G_{n}(x, y), G_{m}(x, y)\right) \leq \varepsilon .
\end{aligned}
$$

It follows that $\left\{\varphi_{n}(x, u, y)\right\}$ and $\left\{\psi_{n}(x, u, y)\right\}$ are both Cauchy sequences in $Z$ for any fixed $(x, u, y) \in X \times X \times Y$ and $(x, v, y) \in X \times Y \times Y$, respectively. Moreover, we know that $\left\{S_{n}(x, y)\right\},\left\{G_{n}(x, y)\right\}$ are both Cauchy sequences in $K(X)$ and $K(Y)$ for any fixed $(x, y) \in X \times Y$, respectively.

(I) Since $Z$ is a complete space, there exists $z \in Z$ such that $\lim _{n \rightarrow \infty} \varphi_{n}(x, u, y)=z$. Let $\varphi(x, u, y):=z$. We show that $\varphi$ is $C_{1}$-upper semicontinuous and

$$
\sup _{(x, u, y) \in X \times Y \times Y}\|\varphi(x, u, y)\|<+\infty .
$$

For any open neighborhood $V$ of zero in $Z$, since $\varphi_{n}$ is $C_{1}$ upper semicontinuous, there exists an open neighborhood $U$ of $(x, u, y)$ such that

$$
\begin{array}{r}
\varphi_{n}\left(x^{\prime}, u^{\prime}, y^{\prime}\right) \in \varphi_{n}(x, u, y)+\frac{V}{3}-C_{1}, \\
\forall\left(x^{\prime}, u^{\prime}, y^{\prime}\right) \in U .
\end{array}
$$

Since $\lim _{n \rightarrow \infty} \varphi_{n}(x, u, y)=\varphi(x, u, y)$, for $n$ large enough, one has

$$
\begin{gathered}
\varphi\left(x^{\prime}, u^{\prime}, y^{\prime}\right) \in \varphi_{n}\left(x^{\prime}, u^{\prime}, y^{\prime}\right)+\frac{V}{3}, \\
\varphi_{n}(x, u, y) \in \varphi(x, u, y)+\frac{V}{3} .
\end{gathered}
$$

It follows from (43) and (44) that

$$
\varphi\left(x^{\prime}, u^{\prime}, y^{\prime}\right) \in \varphi(x, u, y)+V-C_{1}, \quad \forall\left(x^{\prime}, u^{\prime}, y^{\prime}\right) \in U
$$

and so $\varphi$ is $C_{1}$-upper semicontinuous.

On the other hand, $\lambda_{n} \in \Lambda$ shows that

$$
\sup _{(x, u, y) \in X \times X \times Y}\left\|\varphi_{n}(x, u, y)\right\|<+\infty .
$$

Since $\lim _{n \rightarrow \infty} \varphi_{n}(x, u, y)=\varphi(x, u, y)$, it is easy to see that

$$
\sup _{(x, u, y) \in X \times X \times Y}\|\varphi(x, u, y)\|<+\infty .
$$

Moreover, from the fact that $\lim _{n \rightarrow \infty} \varphi_{n}(x, u, y)=$ $\varphi(x, u, y)$ and $\varphi_{n}(x, x, y)=0$, we know that $\varphi(x, x, y)=0$.

(II) Similar to the proof of (I), we know that there exists a $C_{2}$-upper semicontinuous mapping $\psi$ such that $\lim _{n \rightarrow \infty} \psi_{n}(x, u, y)=\psi(x, u, y)$ with $\psi(x, y, y)=0$ and

$$
\sup _{(x, u, y) \in X \times Y \times Y}\|\psi(x, u, y)\|<+\infty .
$$

(III) Since $S_{n}(x, y), G_{n}(x, y)$ are both Cauchy sequences, $K(X)$ and $K(Y)$ are complete, we know that there exist sets $M \in K(X)$ and $N \in K(Y)$ such that

$$
\lim _{n \rightarrow \infty} S_{n}(x, y)=M, \quad \lim _{n \rightarrow \infty} G_{n}(x, y)=N
$$

Let $S(x, y):=M$ and $G(x, y):=N$. Then the fact that $S_{n}$ and $G_{n}$ are both continuous with compact set-values shows that $S$ and $G$ are both continuous with compact set-values.

(IV) We prove that there exist $x \in S(x, y)$ and $y \in G(x, y)$ such that

$$
\begin{aligned}
& \varphi(x, u, y) \notin-\operatorname{int} C_{1}, \quad \forall u \in S(x, y), \\
& \psi(x, v, y) \notin-\operatorname{int} C_{2}, \quad \forall v \in G(x, y) .
\end{aligned}
$$

In fact, since $\left(\varphi_{n}, \psi_{n}, S_{n}, G_{n}\right) \in \Lambda$, there exist sequences $\left\{x_{n}\right\}$ and $\left\{y_{n}\right\}$ such that $x_{n} \in S_{n}\left(x_{n}, y_{n}\right), y_{n} \in G_{n}\left(x_{n}, y_{n}\right)$, and

$$
\varphi_{n}\left(x_{n}, u, y_{n}\right) \notin-\operatorname{int} C_{1}, \quad \forall u \in S_{n}\left(x_{n}, y_{n}\right)
$$

with

$$
\psi\left(x_{n}, v, y_{n}\right) \notin-\operatorname{int} C_{2}, \quad \forall v \in G\left(x_{n}, y_{n}\right) .
$$

Since $X$ and $Y$ are compact, there exist subsequences $\left\{x_{n_{k}}\right\} \subset$ $\left\{x_{n}\right\}$ and $\left\{y_{n_{k}}\right\} \subset\left\{y_{n}\right\}$ such that $x_{n_{k}} \rightarrow x \in X$ and $y_{n_{k}} \rightarrow y \in$ $Y$. From the continuities of $S$ and $G$, we have

$$
\begin{aligned}
& H_{1}\left(S\left(x_{n_{k}}, y_{n_{k}}\right), S(x, y)\right) \longrightarrow 0, \\
& H_{2}\left(G\left(x_{n_{k}}, y_{n_{k}}\right), G(x, y)\right) \longrightarrow 0 .
\end{aligned}
$$

When $n, m$ are large enough, one has

$$
\sup _{(x, y) \in X \times Y} H_{1}\left(S_{n}(x, y), S_{m}(x, y)\right) \leq \varepsilon
$$

and so

$$
H_{1}\left(S_{n_{k}}\left(x_{n_{k}}, y_{n_{k}}\right), S\left(x_{n_{k}}, y_{n_{k}}\right)\right) \leq \varepsilon
$$


holds for $n_{k}$ large enough. By the definition of the Hausdorff metric, we have

$$
\begin{aligned}
d_{1}(x, & S(x, y)) \\
\leq & d_{1}\left(x, x_{n_{k}}\right)+d_{1}\left(x_{n_{k}}, S_{n_{k}}\left(x_{n_{k}}, y_{n_{k}}\right)\right) \\
& +H_{1}\left(S_{n_{k}}\left(x_{n_{k}}, y_{n_{k}}\right), S(x, y)\right) \\
= & d_{1}\left(x, x_{n_{k}}\right) \\
& +H_{1}\left(S_{n_{k}}\left(x_{n_{k}}, y_{n_{k}}\right), S(x, y)\right) \\
\leq & d_{1}\left(x, x_{n_{k}}\right) \\
& +H_{1}\left(S_{n_{k}}\left(x_{n_{k}}, y_{n_{k}}\right), S\left(x_{n_{k}}, y_{n_{k}}\right)\right) \\
& +H_{1}\left(S\left(x_{n_{k}}, y_{n_{k}}\right), S(x, y)\right) .
\end{aligned}
$$

This together with (55) shows that $d_{1}(x, S(x, y))=0$ and so $x \in S(x, y)$. Similarly, we can get $y \in G(x, y)$.

Next we prove that

$$
\varphi(x, u, y) \notin-\operatorname{int} C_{1}, \quad \forall u \in S(x, y) .
$$

By contradiction, we assume that there exists $u_{0} \in S(x, y)$ such that

$$
\varphi\left(x, u_{0}, y\right) \in-\operatorname{int} C_{1} .
$$

Then there exists an open neighborhood $V$ of zero in $Z$ such that

$$
\varphi\left(x, u_{0}, y\right)+V \in-\operatorname{int} C_{1} .
$$

By Lemma 5, there is a sequence $\left\{u_{n_{k}}\right\}$ with $u_{n_{k}} \in S_{n_{k}}\left(x_{n_{k}}, y_{n_{k}}\right)$ such that $u_{n_{k}} \rightarrow u_{0}$. Since $\varphi_{n_{k}}$ is $C_{1}$-upper semicontinuous and $\varphi_{n_{k}}\left(x, u_{0}, y\right) \rightarrow \varphi\left(x, u_{0}, y\right)$, we have

$$
\begin{gathered}
\varphi_{n_{k}}\left(x_{n_{k}}, u_{n_{k}}, y_{n_{k}}\right) \in \varphi_{n_{k}}\left(x, u_{0}, y\right)+\frac{V}{2}-C_{1}, \\
\varphi_{n_{k}}\left(x, u_{0}, y\right) \in \varphi\left(x, u_{0}, y\right)+\frac{V}{2} .
\end{gathered}
$$

From (59) and (60), we get

$$
\varphi_{n_{k}}\left(x_{n_{k}}, u_{n_{k}}, y_{n_{k}}\right) \in-C_{1}-\operatorname{int} C_{1} \subset-\operatorname{int} C_{1} \text {, }
$$

which is in contradiction with the fact that $\varphi_{n_{k}}\left(x_{n_{k}}, u, y_{n_{k}}\right) \notin$ - int $C_{1}$ for all $u \in S_{n_{k}}\left(x_{n_{k}}, y_{n_{k}}\right)$.

Similarly, we can show that $y \in G(x, y)$ satisfies

$$
\psi(x, v, y) \notin-\operatorname{int} C_{2}, \quad \forall v \in G(x, y) .
$$

(V) Let $\lambda=(\varphi, \psi, S, G)$. Then $\lambda \in \Lambda$ and $\lambda_{n} \rightarrow \lambda$. Therefore, $(\Lambda, \rho)$ is a complete metric space. This completes the proof.

Lemma 18. $f: \Lambda \rightrightarrows X \times Y$ is an usco mapping.
Proof. It is easy to see that $f(\lambda)$ is closed for any given $\lambda \in \Lambda$. In fact, for any

$$
\begin{aligned}
& \left(x_{n}, y_{n}\right) \in f(\lambda) \\
& \quad=\{(x, y) \in X \times Y: x \in S(x, y), y \in G(x, y)\}
\end{aligned}
$$

with $x_{n} \rightarrow x$ and $y_{n} \rightarrow y$, we have $x_{n} \in S\left(x_{n}, y_{n}\right)$ and $y_{n} \in G\left(x_{n}, y_{n}\right)$ and so

$$
\begin{aligned}
d_{1}(x, S(x, y)) & \leq d_{1}\left(x, x_{n}\right)+d_{1}\left(x_{n}, S(x, y)\right) \\
& \leq d_{1}\left(x, x_{n}\right)+H_{1}\left(S\left(x_{n}, y_{n}\right), S(x, y)\right) .
\end{aligned}
$$

It follows from the continuity of $S$ that $d_{1}(x, S(x, y))=0$ and so $x \in S(x, y)$. Similarly, we can get $y \in G(x, y)$. Thus, $(x, y) \in f(\lambda)$ and so $f(\lambda)$ is closed. Since $X \times Y$ is compact, we know that $f(\lambda)$ is compact for any given $\lambda \in \Lambda$. In order to show that $f$ is an u.s.c. mapping, from Lemma 4 , it is sufficient to show that $\operatorname{Graph}(f)=\{(\lambda,(x, y)):(x, y) \in$ $f(\lambda)\}$ is closed.

Let $\left(\lambda_{n},\left(x_{n}, y_{n}\right)\right) \in \operatorname{Graph}(f)$ with $\left(\lambda_{n},\left(x_{n}, y_{n}\right)\right) \rightarrow$ $(\lambda,(x, y))$. Then the completeness of $(\Lambda, \rho)$ shows that $\lambda \in \Lambda$. Moreover, we have $x_{n} \in S_{n}\left(x_{n}, y_{n}\right)$ and $y_{n} \in G_{n}\left(x_{n}, y_{n}\right)$ and

$$
\begin{array}{r}
H_{1}\left(S_{n}\left(x_{n}, y_{n}\right), S\left(x_{n}, y_{n}\right)\right) \longrightarrow 0, \\
H_{2}\left(G_{n}\left(x_{n}, y_{n}\right), G\left(x_{n}, y_{n}\right)\right) \longrightarrow 0, \\
H_{1}\left(S\left(x_{n}, y_{n}\right), S(x, y)\right) \longrightarrow 0, \\
H_{2}\left(G\left(x_{n}, y_{n}\right), G(x, y)\right) \longrightarrow 0 .
\end{array}
$$

By the definition of the Hausdorff metric, it follows that

$$
\begin{aligned}
d_{1}(x, S(x, y)) \leq & d_{1}\left(x, x_{n}\right)+d_{1}\left(x_{n}, S_{n}\left(x_{n}, y_{n}\right)\right) \\
& +H_{1}\left(S_{n}\left(x_{n}, y_{n}\right), S\left(x_{n}, y_{n}\right)\right) \\
& +H_{1}\left(S\left(x_{n}, y_{n}\right), S(x, y)\right) \longrightarrow 0, \\
d_{2}(y, G(x, y)) \leq & d_{2}\left(y, y_{n}\right)+d_{2}\left(y_{n}, G_{n}\left(x_{n}, y_{n}\right)\right) \\
& +H_{2}\left(G_{n}\left(x_{n}, y_{n}\right), G\left(x_{n}, y_{n}\right)\right) \\
& +H_{2}\left(G\left(x_{n}, y_{n}\right), G(x, y)\right) \longrightarrow 0 .
\end{aligned}
$$

From (66), we have $(\lambda,(x, y)) \in \operatorname{Graph}(f)$. This completes the proof.

Lemma 19. For any $(\lambda,(x, y)) \in \Lambda \times X \times Y$, the rationality function $\Phi$ is lower semicontinuous at $(\lambda,(x, y))$.

Proof. For any given $r \in R=(-\infty$, $+\infty)$, let

$$
M=\{(\lambda,(x, y)) \in \Lambda \times X \times Y: \Phi(\lambda,(x, y)) \leq r\} .
$$

In order to show $\Phi(\lambda,(x, y))$ is lower semicontinuous, we only need to show that $M$ is closed. Let $\left(\lambda_{n},\left(x_{n}, y_{n}\right)\right) \in M$ with $\left(\lambda_{n},\left(x_{n}, y_{n}\right)\right) \rightarrow(\lambda,(x, y))$. We show that $(\lambda,(x, y)) \in$ $M$, that is, $\Phi(\lambda,(x, y)) \leq r$, which is equivalent to

$$
\max \left\{\sup _{u \in S(x, y)}-\xi_{e_{1}} \varphi(x, u, y), \sup _{v \in G(x, y)}-\xi_{e_{2}} \psi(x, v, y)\right\} \leq r,
$$


where $e_{1} \in \operatorname{int} C_{1}$ and $e_{2} \in \operatorname{int} C_{2}$, that is,

$$
\begin{aligned}
& \xi_{e_{1}} \varphi(x, u, y) \geq-r, \quad \forall u \in S(x, y), \\
& \xi_{e_{2}} \psi(x, v, y) \geq-r, \quad \forall v \in G(x, y),
\end{aligned}
$$

which is equivalent to (by Lemma 7)

$$
\begin{aligned}
& \varphi(x, u, y) \notin-r e_{1}-\operatorname{int} C_{1}, \quad \forall u \in S(x, y), \\
& \psi(x, v, y) \notin-r e_{2}-\operatorname{int} C_{2}, \quad \forall v \in G(x, y) .
\end{aligned}
$$

By way of contradiction, assume there exists $u_{0} \in S(x, y)$ or $v_{0} \in G(x, y)$ such that

$$
\varphi\left(x, u_{0}, y\right) \in-r e_{1}-\operatorname{int} C_{1}
$$

or

$$
\psi\left(x, v_{0}, y\right) \in-r e_{2}-\operatorname{int} C_{2} .
$$

Without loss of generality, assume that (71) holds. Then there exists a neighborhood $V$ of zero in $Z$ such that

$$
\varphi\left(x, u_{0}, y\right)+V \subset-r e_{1}-\operatorname{int} C_{1} .
$$

For $u_{0} \in S(x, y)$, since $S(x, y)$ and $S_{n}\left(x_{n}, y_{n}\right)$ are compact with $S_{n}\left(x_{n}, y_{n}\right) \rightarrow S(x, y)$, by Lemma 5, there exists $u_{n} \in S_{n}\left(x_{n}, y_{n}\right)$ with $u_{n} \rightarrow u_{0}$. Since $\varphi_{n}$ is $C_{1}$-upper semicontinuous and $\varphi_{n}\left(x, u_{0}, y\right) \rightarrow \varphi\left(x, u_{0}, y\right)$, we have

$$
\begin{gathered}
\varphi_{n}\left(x_{n}, u_{n}, y_{n}\right) \in \varphi_{n}\left(x, u_{0}, y\right)+\frac{V}{2}-C_{1}, \\
\varphi_{n}\left(x, u_{0}, y\right) \in \varphi\left(x, u_{0}, y\right)+\frac{V}{2} .
\end{gathered}
$$

It follows from (73) and (74) that

$$
\varphi_{n}\left(x_{n}, u_{n}, y_{n}\right) \in-r e_{1}-\operatorname{int} C_{1} .
$$

On the other hand, since $\left(\lambda_{n},\left(x_{n}, y_{n}\right)\right) \in M$, we know that $\Phi\left(\lambda_{n},\left(x_{n}, y_{n}\right)\right) \leq r$ and so

$$
\begin{aligned}
\max \left\{\sup _{u \in S_{n}\left(x_{n}, y_{n}\right)}-\xi_{e_{1}} \varphi_{n}\left(x_{n}, u, y_{n}\right),\right. \\
\left.\sup _{v \in G_{n}\left(x_{n}, y_{n}\right)}-\xi_{e_{2}} \psi_{n}\left(x_{n}, v, y_{n}\right)\right\} \leq r .
\end{aligned}
$$

It follows that

$$
\xi_{e_{1}} \varphi_{n}\left(x_{n}, u, y_{n}\right) \geq-r, \quad \forall u \in S_{n}\left(x_{n}, y_{n}\right)
$$

Now Lemma 10 implies that

$$
\varphi_{n}\left(x_{n}, u, y_{n}\right) \notin-r e_{1}-\operatorname{int} C_{1}, \quad \forall u \in S_{n}\left(x_{n}, y_{n}\right),
$$

which is in contradiction with (75). Thus, we know that $\Phi(\lambda,(x, y))$ is lower semicontinuous. This completes the proof.
Theorem 20. For all $\lambda \in \Lambda$, the problem $\lambda$ is generalized wellposed. Moreover, for all $\lambda \in \Lambda$, if $E(\lambda)=\{(x, y)\}$ (a singleton), then the problem $\lambda$ is well-posed.

Proof. Let $\lambda_{n} \in \Lambda$ with $\lambda_{n} \rightarrow \lambda$ and $\left(x_{n}, y_{n}\right) \in E\left(\lambda_{n}, \varepsilon_{n}\right)$ with $\varepsilon_{n} \rightarrow 0$. Then

$$
\begin{gathered}
d\left(\left(x_{n}, y_{n}\right), f\left(\lambda_{n}\right)\right) \leq \varepsilon_{n}, \\
\Phi\left(\lambda_{n},\left(x_{n}, y_{n}\right)\right) \leq \varepsilon_{n} .
\end{gathered}
$$

By (79), there exists $\left(\bar{x}_{n}, \bar{y}_{n}\right) \in f\left(\lambda_{n}\right)$ such that $d\left(\left(x_{n}, y_{n}\right)\right.$, $\left.\left(\bar{x}_{n}, \bar{y}_{n}\right)\right) \rightarrow 0$. Since $f$ is an usco mapping, by Lemma 6 , there exists a subsequence $\left\{\left(\bar{x}_{n_{k}}, \bar{y}_{n_{k}}\right)\right\} \subset\left\{\left(\bar{x}_{n}, \bar{y}_{n}\right)\right\}$ such that $\left(\bar{x}_{n_{k}}, \bar{y}_{n_{k}}\right) \rightarrow(x, y) \in f(\lambda)$. It follows that

$$
\begin{aligned}
& d\left(\left(x_{n_{k}}, y_{n_{k}}\right),(x, y)\right) \\
& \leq d\left(\left(x_{n_{k}}, y_{n_{k}}\right),\left(\bar{x}_{n_{k}}, \bar{y}_{n_{k}}\right)\right) \\
& \quad+d\left(\left(\bar{x}_{n_{k}}, \bar{y}_{n_{k}}\right),(x, y)\right) \longrightarrow 0
\end{aligned}
$$

and so

$$
\left(x_{n_{k}}, y_{n_{k}}\right) \longrightarrow(x, y) \in f(\lambda) .
$$

On the other hand, the lower semicontinuity of $\Phi$ shows that

$$
\begin{aligned}
0 \leq \Phi(\lambda,(x, y)) & \leq \liminf \Phi\left(\lambda_{n},\left(x_{n_{k}}, y_{n_{k}}\right)\right) \\
& \leq \liminf \varepsilon_{n}=0
\end{aligned}
$$

which implies that $\Phi(\lambda,(x, y))=0$. It follows from (82) and (83) that $(x, y) \in E(\lambda)$ and so the problem $\lambda$ is generalized well-posed.

Moreover, we show that, if $E(\lambda)=\{(x, y)\}$, then the problem $\lambda$ is well-posed. If the sequence $\left\{\left(x_{n}, y_{n}\right)\right\}$ does not converge to $(x, y)$, then there exist an open neighborhood $O$ of $(x, y)$ and a subsequence $\left\{\left(x_{n_{k}}, y_{n_{k}}\right)\right\}$ of $\left\{\left(x_{n}, y_{n}\right)\right\}$ such that $\left(x_{n_{k}}, y_{n_{k}}\right) \notin O$. By the proof of the first part, we know that $\left(x_{n_{k}}, y_{n_{k}}\right) \rightarrow(x, y)$. This is in contradiction with $\left(x_{n_{k}}, y_{n_{k}}\right) \notin$ $O$. This completes the proof.

Example 21. Let $X=Y=[0,2] \times[0,2], Z=(-\infty,+\infty) \times$ $(-\infty,+\infty), C=[0,+\infty) \times[0,+\infty)$, and $e=(1,1)$. Let

$$
\begin{gathered}
S(x, y)=G(x, y)=[0,1] \times[0,1], \quad \forall(x, y) \in X \times Y, \\
\varphi(x, u, y)=x-u, \quad \psi(x, v, y)=y-v, \\
\forall x, u \in X, \quad \forall y, v \in Y .
\end{gathered}
$$

Then it is easy to see that $\lambda=(\varphi, \psi, S, G) \in \Lambda$ and

$$
f(\lambda)=\{(x, y) \mid x \in[0,1] \times[0,1], y \in[0,1] \times[0,1]\}
$$

with

$$
\begin{gathered}
E(\lambda)=\left\{(x, y) \in f(\lambda) \mid x=\left(x_{1}, x_{2}\right), y=\left(y_{1}, y_{2}\right),\right. \\
\left.\max \left\{x_{1}, x_{2}\right\}=1, \max \left\{y_{1}, y_{2}\right\}=1\right\} .
\end{gathered}
$$

Furthermore, by Theorem 20, the problem $\lambda$ is generalized well-posedness. 
Remark 22. When $\varphi=\psi$ and $S=G$, the well-posedness for the problem $\lambda$ was studied by [20]. Theorem 20 presented in this paper can be considered as a generalization and extension of Theorem 4.1 in [20].

From Theorem 20, we have the following corollaries.

Corollary 23. For all $\lambda \in \Lambda$, the problem $\lambda$ is (generalized) Hadamard well-posed.

Corollary 24. For all $\lambda \in \Lambda$, the problem $\lambda$ is (generalized) $L P$ well-posed.

Remark 25. When $\varphi=\psi, S=G$, the generalized Hadamard well-posedness for the problem $\lambda$ was studied by [16]. Corollary 23 presented in this paper generalizes and extends Theorem 4.2 in [16].

Remark 26. We note that, when

$$
\begin{aligned}
& \varphi(x, u, y)=f(u, y)-f(x, y), \\
& \psi(x, v, y)=g(x, v)-g(x, y),
\end{aligned}
$$

the generalized LP well-posedness for the symmetric vector quasi-equilibrium problem was studied by [19].

\section{Conflict of Interests}

The authors declare that there is no conflict of interests regarding the publication of this paper.

\section{Acknowledgments}

The authors would like to thank the editor and the reviewers for their helpful comments and suggestions, which have improved the presentation of the paper. This work was supported by the National Natural Science Foundation of China (11171237).

\section{References}

[1] G.-Y. Chen, X. Huang, and X. Yang, Vector Optimization: SetValued and Variational Analysis, vol. 541 of Lecture Notes in Economics and Mathematical Systems, Springer, Berlin, Germany, 2005.

[2] F. Giannessi, Vector Variational Inequalities and Vector Equilibria: Mathematical Theories, Kluwer Academic Publishers, Dordrecht, The Netherlands, 2000.

[3] D. T. Luc, Theory of Vector Optimization, Lecture Notes in Economics and Mathematical Systems, Springer, Berlin, Germany, 1989.

[4] A. N. Tykhonov, "On the stability of the functional optimization problem," USSR Journal of Computational Mathematics and Mathematical Physics, vol. 6, pp. 631-634, 1996.

[5] R. Lucchetti and F. Patrone, "A characterization of Tyhonov well-posedness for minimum problems, with applications to variational inequalities," Numerical Functional Analysis and Optimization, vol. 3, no. 4, pp. 461-476, 1981.

[6] M. B. Lignola and J. Morgan, "Well-posedness for optimization problems with constraints defined by variational inequalities having a unique solution," Journal of Global Optimization, vol. 16, no. 1, pp. 57-67, 2000.

[7] Y.-P. Fang, N.-J. Huang, and R. Hu, "Well-posedness for equilibrium problems and for optimization problems with equilibrium constraints," Computers \& Mathematics with Applications, vol. 55, no. 1, pp. 89-100, 2008.

[8] R. Hu, Y. P. Fang, N. J. Huang, and M. M. Wong, "Wellposedness of systems of equilibrium problems," Taiwanese Journal of Mathematics, vol. 14, no. 6, pp. 2435-2446, 2010.

[9] H. Yang and J. Yu, "Unified approaches to well-posedness with some applications," Journal of Global Optimization, vol. 31, no. 3, pp. 371-381, 2005.

[10] R. Lucchetti and J. P. Revalski, Recent Development in Well-Posed Variational Problems, Kluwer Academic Publishers, Dordrecht, The Netherlands, 1995.

[11] X. X. Huang, X. Q. Yang, and D. L. Zhu, "Levitin-Polyak wellposedness of variational inequality problems with functional constraints," Journal of Global Optimization, vol. 44, no. 2, pp. 159-174, 2009.

[12] S. J. Li and M. H. Li, "Levitin-Polyak well-posedness of vector equilibrium problems," Mathematical Methods of Operations Research, vol. 69, no. 1, pp. 125-140, 2009.

[13] M. H. Li, S. J. Li, and W. Y. Zhang, "Levitin-Polyak wellposedness of generalized vector quasi-equilibrium problems," Journal of Industrial and Management Optimization, vol. 5, no. 4, pp. 683-696, 2009.

[14] J.-W. Peng, S.-Y. Wu, and Y. Wang, "Levitin-Polyak wellposedness of generalized vector equilibrium problems," Taiwanese Journal of Mathematics, vol. 15, no. 5, pp. 2311-2330, 2011.

[15] J. Salamon, "Closedness and Hadamard well-posedness of the solution map for parametric vector equilibrium problems," Journal of Global Optimization, vol. 47, no. 2, pp. 173-183, 2010.

[16] D. T. Peng, J. Yu, and N. H. Xiu, “The uniqueness and wellposedness of vector equilibrium problems with a representation theorem for the solution set," Fixed Point Theory and Applications, vol. 2014, article 115, 2014.

[17] S. J. Li and W. Y. Zhang, "Hadamard well-posed vector optimization problems," Journal of Global Optimization, vol. 46, no. 3, pp. 383-393, 2010.

[18] X. J. Long and N. J. Huang, "Metric characterization of $\alpha$-wellposedness for symmetric quasi-equilibrium problems," Journal of Global Optimization, vol. 45, no. 3, pp. 459-471, 2009.

[19] Y. Han and X.-H. Gong, "Levitin-Polyak well-posedness of symmetric vector quasi-equilibrium problems," Optimization, 2014.

[20] X. Deng and S. Xiang, "Well-posed generalized vector equilibrium problems," Journal of Inequalities and Applications, vol. 2014, article 127, 2014.

[21] J.-Y. Fu, "Symmetric vector quasi-equilibrium problems," Journal of Mathematical Analysis and Applications, vol. 285, no. 2, pp. 708-713, 2003.

[22] J. P. Aubin and A. Cellina, Differential Inclusions, vol. 264, Springer, New York, NY, USA, 1984.

[23] C. Berge, Topological Spaces, Oliver and Boyd, Edinburgh, UK, 1963.

[24] J. Yu, "Essential weak efficient solution in multiobjective optimization problems," Journal of Mathematical Analysis and Applications, vol. 166, no. 1, pp. 230-235, 1992. 
[25] J. Yu, H. Yang, and C. Yu, "Structural stability and robustness to bounded rationality for non-compact cases," Journal of Global Optimization, vol. 44, no. 1, pp. 149-157, 2009.

[26] C. Gerth and P. Weidner, "Nonconvex separation theorems and some applications in vector optimization," Journal of Optimization Theory and Applications, vol. 67, no. 2, pp. 297320, 1990. 


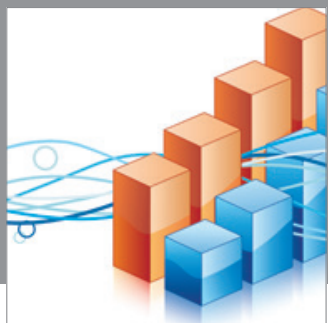

Advances in

Operations Research

mansans

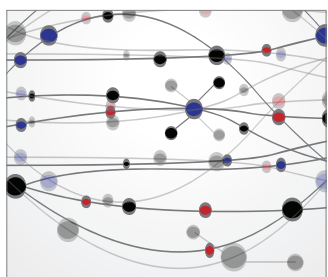

The Scientific World Journal
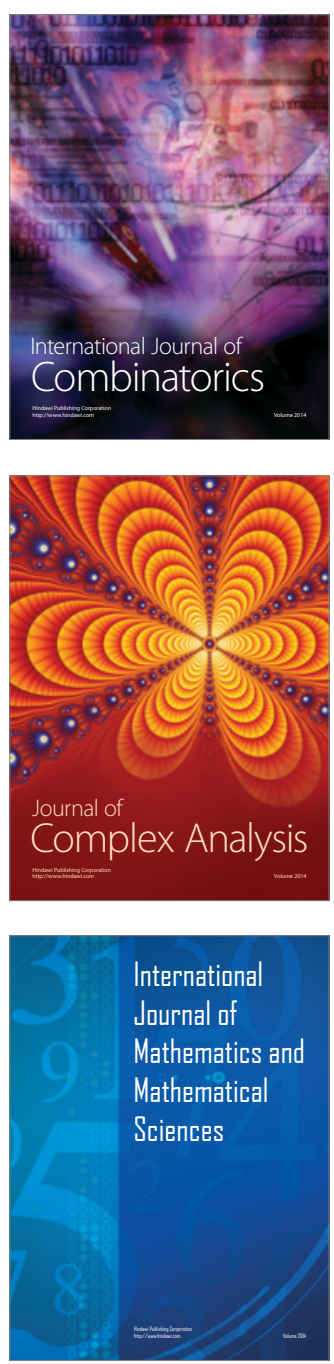
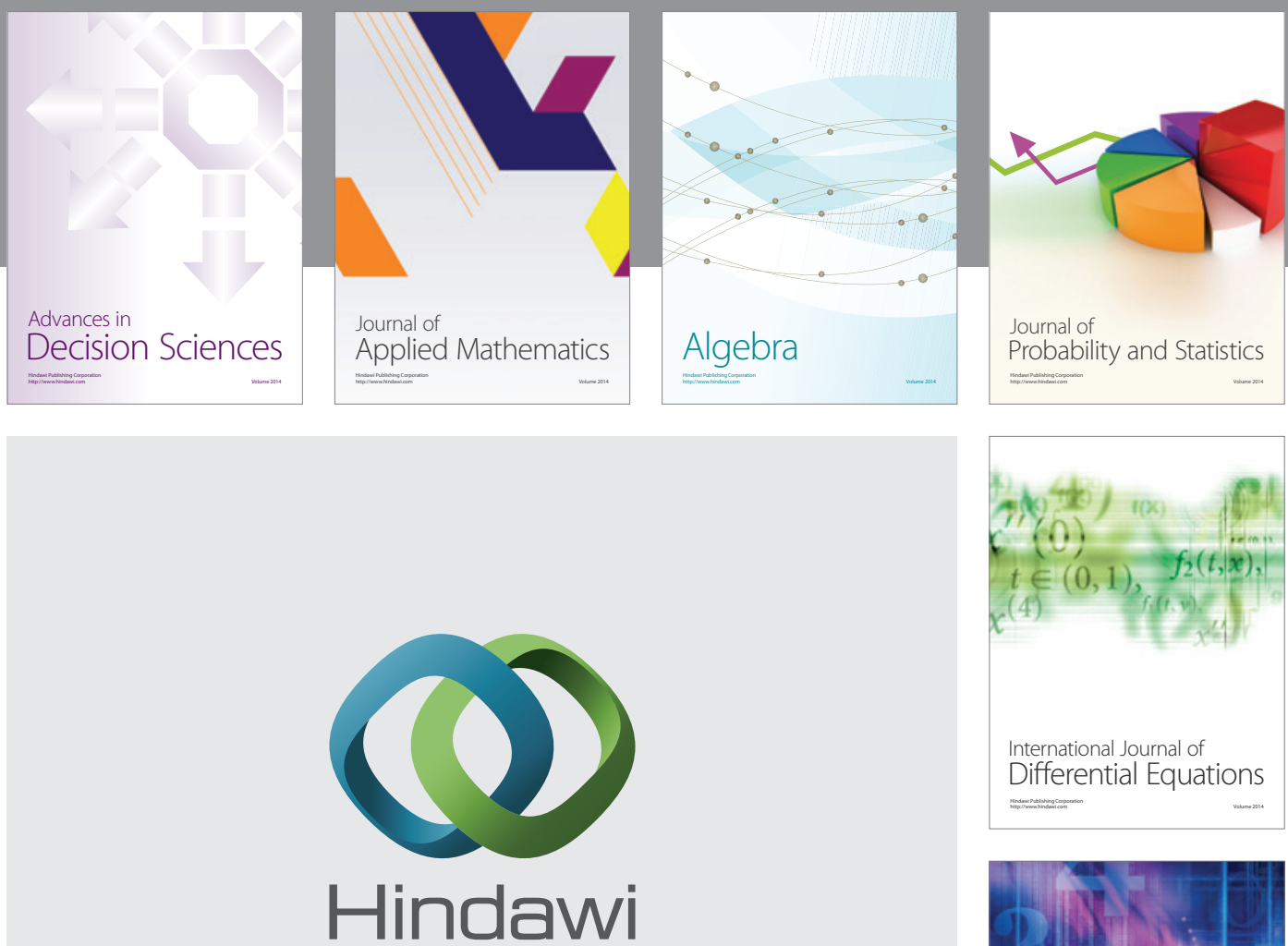

Submit your manuscripts at http://www.hindawi.com
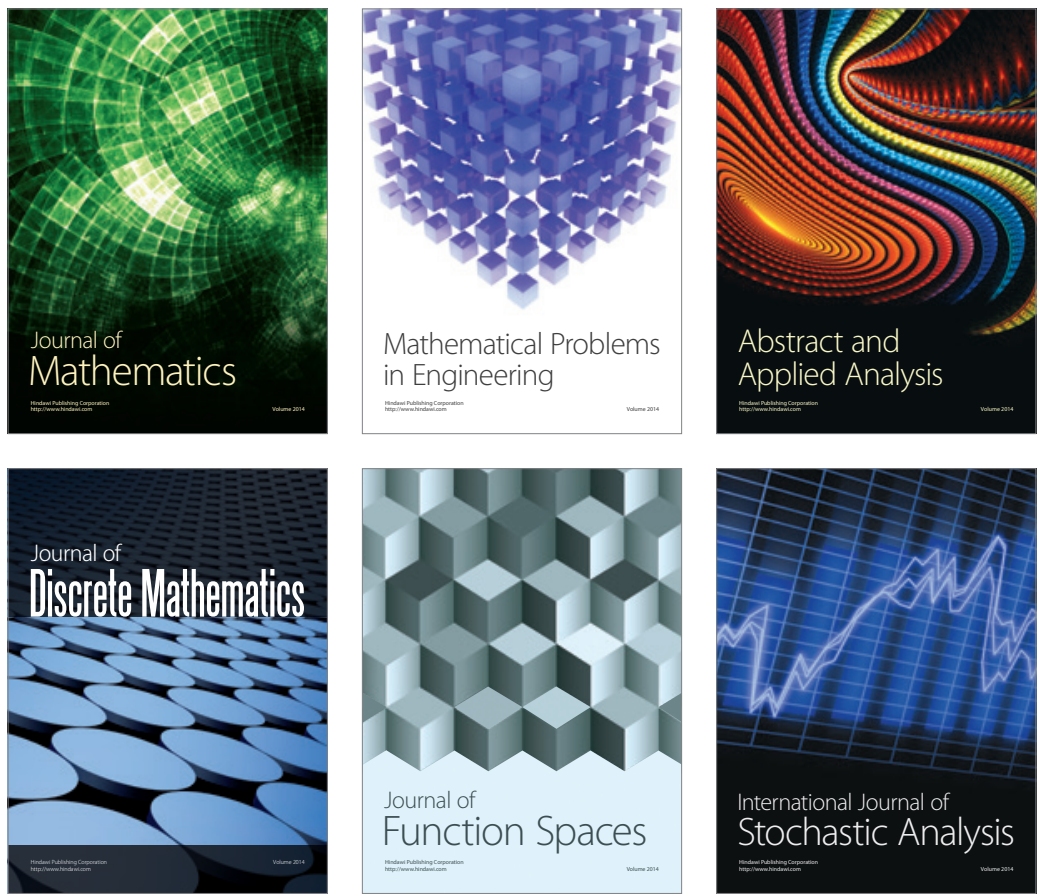

Journal of

Function Spaces

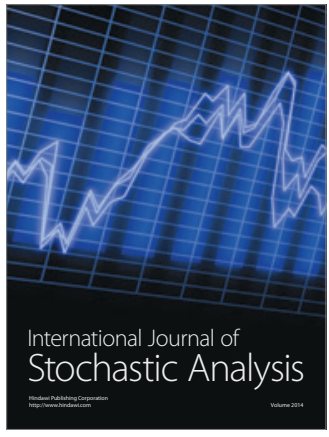

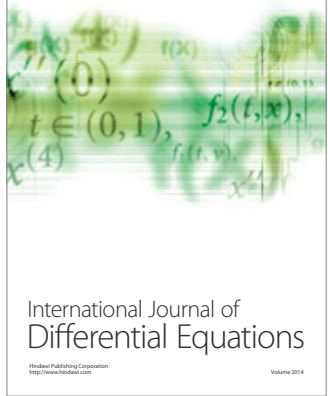
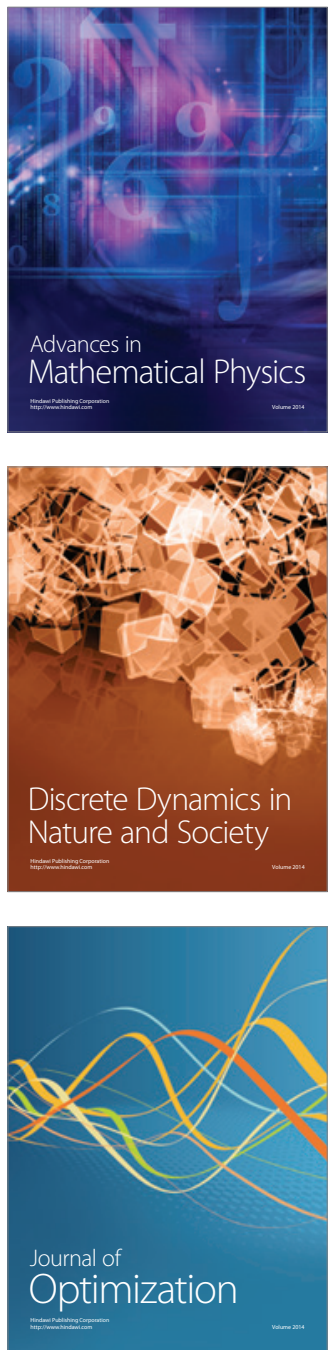